OPEN ACCESS

Edited by:

Dieter Wicher,

Max Planck Institute for Chemical

Ecology, Germany

Reviewed by:

Daniela Marazziti,

Italian National Research Council

(CNR), Italy

Patricia Lagos,

Universidad de la República, Uruguay

${ }^{*}$ Correspondence:

Nicolas F. Berbar

nberbari@iupui.edu

${ }^{\dagger}$ These authors have contributed equally to this work

Specialty section:

This article was submitted to

Cellular Neurophysiology,

a section of the journal

Frontiers in Cellular Neuroscience

Received: 15 March 2019

Accepted: 29 May 2019

Published: 12 June 2019

Citation:

Bansal R, Engle SE, Antonellis PJ, Whitehouse LS, Baucum AJ II,

Cummins TR, Reiter JF and

Berbari NF (2019) Hedgehog Pathway

Activation Alters Ciliary Signaling

in Primary Hypothalamic Cultures.

Front. Cell. Neurosci. 13:266.

doi: 10.3389/fncel.2019.00266

\section{Hedgehog Pathway Activation Alters Ciliary Signaling in Primary Hypothalamic Cultures}

\author{
Ruchi Bansal'1t, Staci E. Engle ${ }^{1+}$, Patrick J. Antonellis ${ }^{1}$, Logan S. Whitehouse ${ }^{1}$, \\ Anthony J. Baucum II,2, Theodore R. Cummins ${ }^{1,2}$, Jeremy F. Reiter ${ }^{3}$ and \\ Nicolas F. Berbari1,2,4*
}

\begin{abstract}
'Department of Biology, Indiana University - Purdue University Indianapolis, Indianapolis, IN, United States, ${ }^{2}$ Stark Neurosciences Research Institute, Indianapolis, IN, United States, ${ }^{3}$ Department of Biochemistry and Biophysics, Cardiovascular Research Institute, University of California, San Francisco, San Francisco, CA, United States, ${ }^{4}$ Center for Diabetes and Metabolic Disorders Research, Indiana University School of Medicine, Indianapolis, IN, United States
\end{abstract}

Primary cilia dysfunction has been associated with hyperphagia and obesity in both ciliopathy patients and mouse models of cilia perturbation. Neurons throughout the brain possess these solitary cellular appendages, including in the feeding centers of the hypothalamus. Several cell biology questions associated with primary neuronal cilia signaling are challenging to address in vivo. Here we utilize primary hypothalamic neuronal cultures to study ciliary signaling in relevant cell types. Importantly, these cultures contain neuronal populations critical for appetite and satiety such as proopiomelanocortin (POMC) and agouti related peptide (AgRP) expressing neurons and are thus useful for studying signaling involved in feeding behavior. Correspondingly, these cultured neurons also display electrophysiological activity and respond to both local and peripheral signals that act on the hypothalamus to influence feeding behaviors, such as leptin and melanin concentrating hormone $(\mathrm{MCH})$. Interestingly, we found that cilia mediated hedgehog signaling, generally associated with developmental processes, can influence ciliary GPCR signaling (Mchr1) in terminally differentiated neurons. Specifically, pharmacological activation of the hedgehog-signaling pathway using the smoothened agonist, SAG, attenuated the ability of neurons to respond to ligands $(\mathrm{MCH})$ of ciliary GPCRs. Understanding how the hedgehog pathway influences cilia GPCR signaling in terminally differentiated neurons could reveal the molecular mechanisms associated with clinical features of ciliopathies, such as hyperphagia-associated obesity.

Keywords: cilia, primary neuronal cultures, hypothalamus, leptin, melanin concentrating hormone receptor 1 , hedgehog signaling, smoothened, SAG

\section{INTRODUCTION}

The hypothalamus is a complex brain region consisting of several nuclei that regulate food and water intake, circadian rhythm, sexual behavior, and body temperature. Two hypothalamic nuclei involved in feeding behavior, the arcuate nucleus (ARC) and the paraventricular nucleus (PVN), contain several neuronal populations that respond to neuropeptides, and hormones produced both centrally and peripherally (Schwartz et al., 2000; Andermann and Lowell, 2017). Primary neuronal cultures are a valuable tool to help better understand the cell biology of how hypothalamic neurons respond to these stimuli and ultimately regulate feeding behaviors. For example, the 
mechanisms underlying genetic disorders associated with hyperphagia and obesity, such as Bardet-Biedl syndrome (BBS) and Alström syndrome (ALMS) remain unclear. Both BBS and ALMS are classified as ciliopathies, as primary cilia dysfunction is thought to be the cellular etiology of the disorders (Vaisse et al., 2017). Primary cilia are small microtubule based cellular appendages critical to coordinating diverse signaling pathways on many cell types in nearly every organ system, including neurons of the CNS (Singla and Reiter, 2006; Berbari et al., 2009). Hypothalamic neuronal cilia appear critical to normal feeding behavior. Both conditional mouse models of BBS and cilia loss become hyperphagic and obese (Mykytyn et al., 2004; Davenport et al., 2007; Berbari et al., 2013; Guo et al., 2016). Intriguingly, congenital loss of primary cilia from POMC neurons also results in hyperphagia and obesity (Davenport et al., 2007). How primary cilia on these neurons regulate food intake is poorly understood, but several G-protein coupled receptors (GPCRs) have been found to preferentially localize to primary cilia, including melanin concentrating hormone receptor 1 (Mchr1) and melanocortin receptor 4 (Mc4r), both of which are expressed in the hypothalamus along with their ligands, $\mathrm{MCH}$ and alpha-melanocyte stimulating hormone ( $\alpha-\mathrm{MSH})$, respectively, and have established roles in feeding behavior and obesity (Desy and Pelletier, 1978; Bittencourt et al., 1992; Berbari et al., 2008a; Siljee et al., 2018).

In vitro model systems of primary cilia have helped elucidate the sensory and regulatory role these organelles play in different cell types (Praetorius and Spring, 2001; Rohatgi et al., 2007). Primary cultures of the hippocampus have been useful in characterizing primary cilia in vitro (Berbari et al., 2007; Yao et al., 2015). However, primary hypothalamic cultures have not been as widely used. Here we aim to establish an in vitro system using cultured hypothalamic neurons to understand new aspects of cilia signaling which may impact feeding behavior. We compare primary cultures obtained from the hippocampus to the hypothalamus and show that hypothalamic cultures express significantly higher levels of region-specific genes, such as POMC and AgRP. Moreover, we show primary hypothalamic cultures possess primary cilia and are responsive to both peripheral (leptin) and central (MCH) neuropeptides. For the first time, we demonstrate that the cilia mediated hedgehog pathway agonist, SAG, attenuates normal electrophysiological responses to a ciliary GPCR ligand in terminally differentiated neurons. These data suggest an integration between the hedgehog pathway and ciliary GPCR signaling in terminally differentiated neurons.

\section{RESULTS}

\section{Hypothalamus Derived Cultures Contain Populations of Region-Specific Neurons and Glia}

To determine the utility of hypothalamic neuronal cultures, we compared hypothalamic cultures to hippocampal cultures from the same animals (Figure 1). Similar percentages of both neurons and glia were present, as observed by $\beta$-tubulin III and GFAP staining (Figures 1A,B). To determine if hypothalamic cultures expressed region specific peptides in vitro, we stained for both POMC and $\beta$-endorphin and found more cells expressing these neuropeptides when compared to hippocampal-derived controls (Figures 2A,B). Similar results were observed using quantitative PCR for hypothalamic gene expression (Figure 2C). To further confirm that neurons from multiple hypothalamic regions were present in vitro, we utilized Cre recombinase inducible alleles with a tdTomato fluorescent reporter for both POMC and Mchr1 expressing neurons which are found in the ARC and PVN in vivo and reporter positive cells were observed in vitro (Berglund et al., 2013; Engle et al., 2018; Figure 2D). Together, these results suggest that similar to cultured hippocampal primary neurons, hypothalamus derived neurons display expression patterns like those observed in vivo.

\section{Hypothalamic Neurons in Culture Respond to Leptin}

To determine if cultured neurons are responsive to neuropeptides, we treated them with leptin and assessed phosphorylation, and nuclear localization of STAT3 (pSTAT3) (Banks et al., 2000; Pissios et al., 2003). pSTAT3 was significantly increased after leptin treatment (Figure 3A). To further assess if these neurons were also electrophysiologically active and capable of responding with changes in firing similar to those observed in vivo, we isolated the neurons onto multi-electrode arrays (MEAs) and recorded extracellular potentials (Spira and Hai, 2013). In patch clamp recording experiments, AgRP neurons decrease activity, and POMC neurons increase activity in response to leptin (Cowley et al., 2001). Assessment of our MEA data revealed greater variation in the change in firing rate in response to leptin addition compared to vehicle addition (Figure 3B). We observed predictable responses to leptin. We considered any activity changes twice that of vehicle to be an increase (blue points on Figure $\mathbf{3 B}$ graph) while changes half of the vehicle were considered to be a decrease (red points on Figure 3B graph). Electrode responses between those groups (gray points on Figure 3B) either did not change with leptin or are the result of changes from individual cells canceling each other out in the overall extracellular field potential recorded by that electrode. These results demonstrate that cultured hypothalamic neurons are capable of responding to classical neuropeptides, such as leptin, involved in feeding behavior and energy homeostasis.

\section{Hypothalamic Neurons Possess Cilia in Culture}

To determine if hypothalamic neurons possess primary cilia in vitro, we stained hypothalamic and hippocampal cells with cilia specific markers such as adenylyl cyclase III (ACIII) or ADP Ribosylation Factor Like GTPase 13B (Arl13b) (Bishop et al., 2007; Caspary et al., 2007). Similar to other neuronal culture systems, we observed nearly half of $\beta$-Tubulin III positive neurons possessed cilia (Figure 4A; Berbari et al., 2007). In contrast, only $25 \%$ of GFAP positive astrocytes possessed ACIII positive cilia (Figure 4B). To determine if POMC neurons 

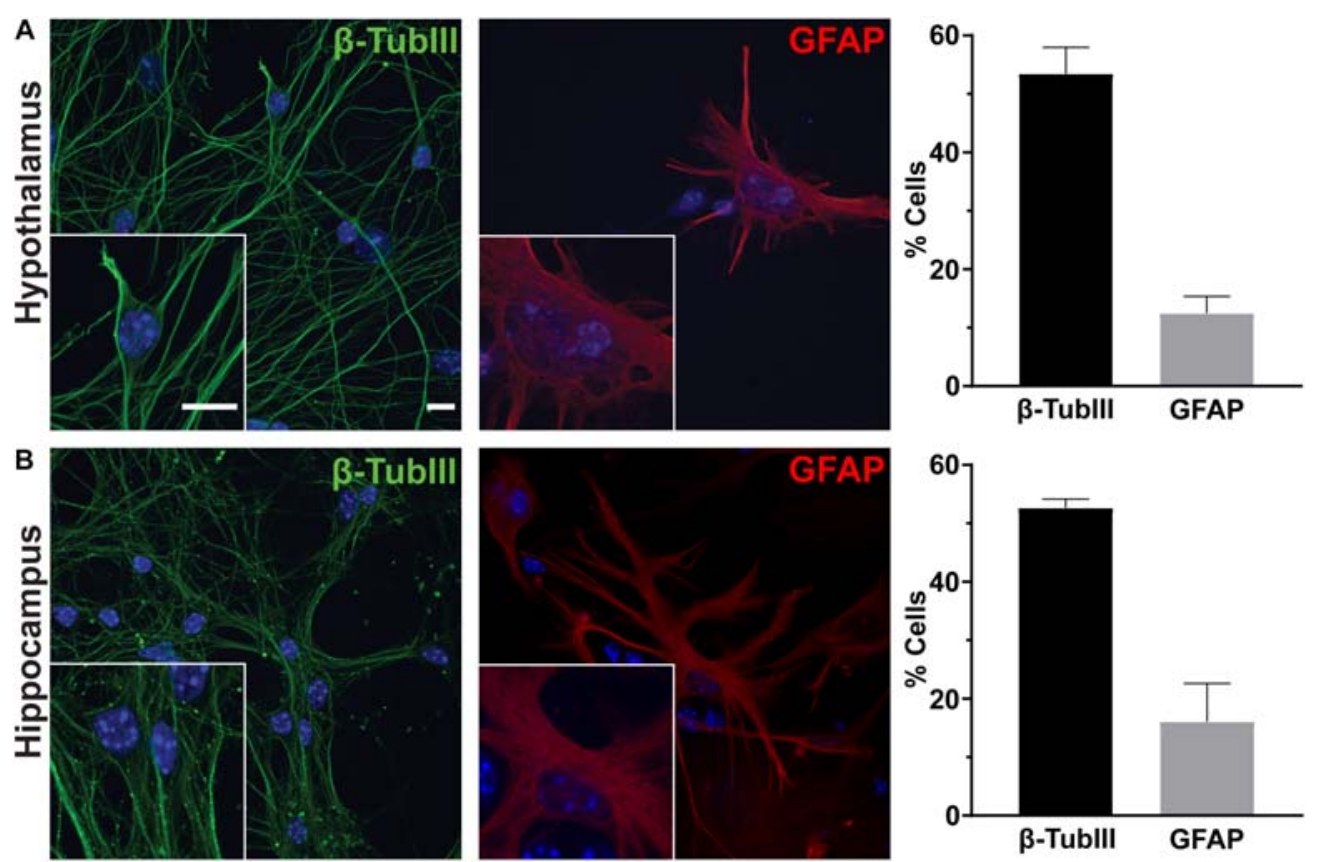

FIGURE 1 | Hypothalamic and hippocampal culture content. Hypothalamic (A) and hippocampal (B) cultures from perinatal mice contain neurons [ $\beta$-tubulin III ( $\beta$-TubIII), green] and glia [glial fibrillary acidic protein (GFAP), red] after 7 days in culture. The percentage of cells positive for the associated stain divided by the total number of cells counted per hypothalamus or hippocampus is graphed as mean \pm SEM. Hoechst stained nuclei are blue. Scale bars are $10 \mu \mathrm{m}$. Data collected from 3 hypothalamic and hippocampal samples from 3 experimental days. $\beta$-tubulin III positive cells/total hypothalamic nuclei: 336/628, GFAP positive cells/total hypothalamic nuclei: 126/647, $\beta$-tubulin III positive cells/total hippocampal nuclei: 323/613, and GFAP positive cells/total hippocampal nuclei: $108 / 617$.

in vitro possess primary cilia, we also labeled these cultures with POMC or $\beta$-endorphin antibodies. We found that nearly $60 \%$ of hypothalamic neurons expressing either POMC or $\beta$-endorphin also possess Arl13b positive cilia (Figures 4C,D). These data confirm that neurons in hypothalamic cultures possess primary cilia, similar to those derived from the hippocampus and nucleus accumbens (Berbari et al., 2007; Yao et al., 2015).

\section{Cilia Mediated Hedgehog Pathway Agonism Impacts Cilia GPCR Mediated Neuronal Activity}

Several GPCRs preferentially localize to primary cilia of neurons throughout the brain, including Mchr1 (Berbari et al., 2008b; Hamamoto et al., 2016; Tomoshige et al., 2017). We observe Mchr1 colocalizing with the cilia marker ACIII (Figure 5A). In olfactory neurons changes in cilia mediated hedgehog signaling altered GPCR localization to cilia (Maurya et al., 2017). To see if activation of the hedgehog pathway affected the number of Mchr1 positive cilia in hypothalamic neurons, we treated cultures with $400 \mathrm{nM}$ smoothened agonist (SAG) for $24 \mathrm{~h}$ and immunolabelled with Mchrl and ACIII. We did not see any change in the percentage of ciliated cells after treatment with SAG nor did we see any difference in the number of Mchrl positive cilia (Figures 5B,C). We next assessed if SAG treatment could impact cilia length or electrophysiological activity. Published data suggests that alterations in neuronal cilia length can influence their ability to signal (Besschetnova et al., 2010; Tomoshige et al., 2017). However, we did not observe cilia length changes with SAG treatment (Figure 5D). Furthermore, SAG treatment alone did not impact the firing rate of hypothalamic cultures (Figure 5E). Interestingly we saw activation of the hedgehog pathway can influence responses to $\mathrm{MCH}$. $\mathrm{MCH}(1 \mu \mathrm{M})$ significantly decreases the firing rate of hypothalamic cultures (Figure 5F), similar to observations made in slice recordings (Gao and van den Pol, 2001). However, MCH elicits no change when cultures were pretreated with SAG (Figure 5F). These results suggest that there may be functional integration between ciliary GPCR signaling and the hedgehog pathway. Future studies will investigate the physiological relevance of persistent hedgehog pathway expression in terminally differentiated hypothalamic neurons as well as the ability for hedgehog signaling to influence other ciliary GPCRs.

\section{DISCUSSION}

The ability to culture primary rodent neurons that establish and maintain their hypothalamic specific identity, responsiveness and activity in vitro has been limited. Here we applied improved culturing techniques and utilized a media developed specifically to enhance neuronal activity in vitro (Bardy et al., 2015). We confirmed that hypothalamic derived neurons expressed hypothalamic genes such as $\beta$-endorphin and that they responded to central ( $\mathrm{MCH})$ and peripheral (leptin) signals with changes in spontaneous firing as occurs in slice recordings 


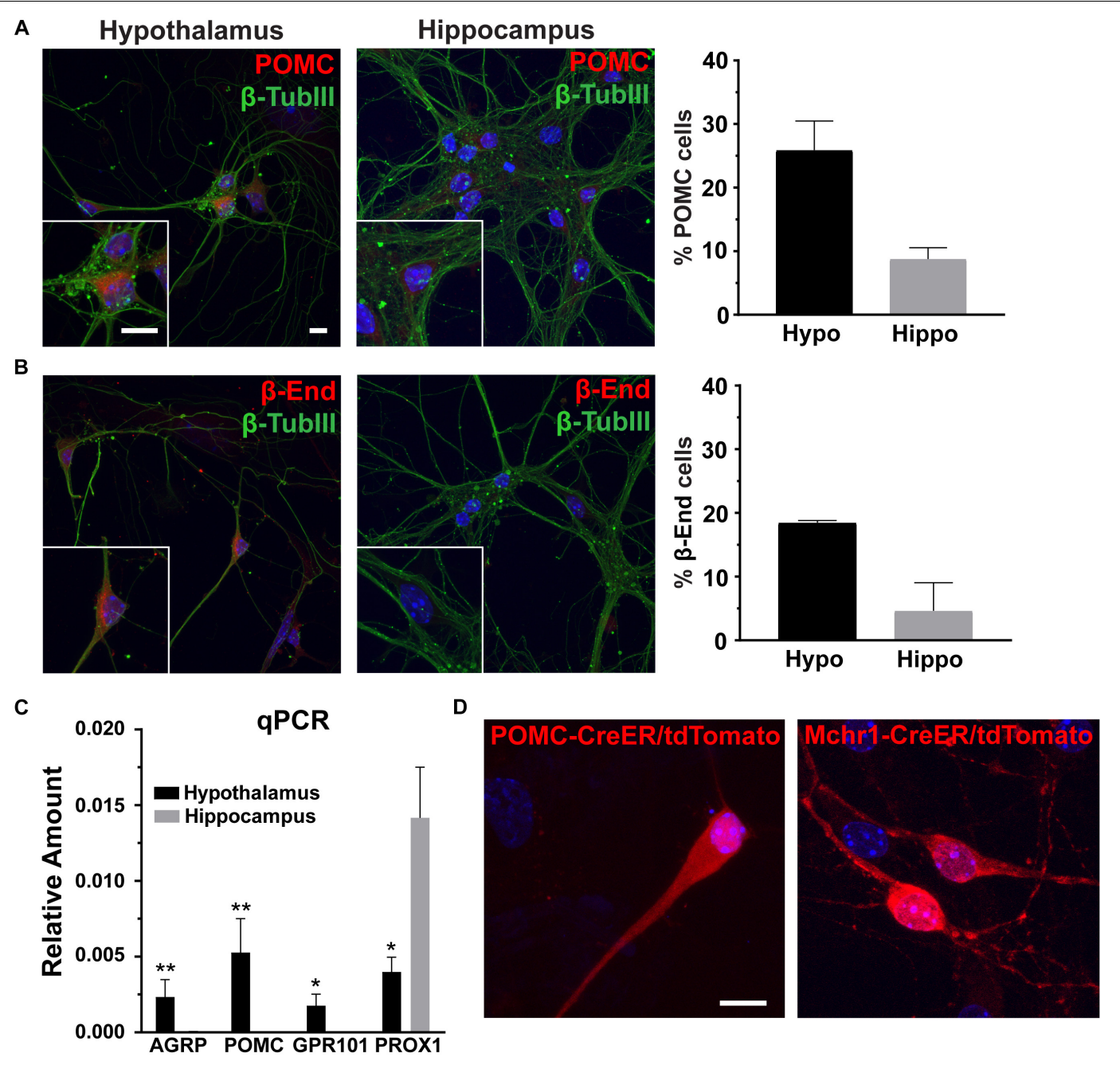

FIGURE 2 | Hypothalamic characteristics in vitro. (A) Proopiomelanocortin (POMC, red) and (B) $\beta$-endorphin ( $\beta$-End, red) staining in hippocampal and hypothalamic cultures co-stained for $\beta$-tubulin III ( $\beta$-Tublll, green). Scale bars are $10 \mu \mathrm{m}$. Percentage of cells positive for POMC or $\beta$-endorphin divided by the total number of cells counted in the hypothalamus and hippocampus graphed as mean \pm SEM. Data collected from 3 hypothalamic and hippocampal samples from 3 experimental days. POMC positive cells/total hypothalamic nuclei: 166/642, $\beta$-endorphin positive cells/total hypothalamic nuclei: 203/657, POMC positive cells/total hippocampal nuclei: 57/653, and $\beta$-endorphin positive cells/total hippocampal nuclei: 69/678. (C) Quantitative PCR (qPCR) analysis on total RNA isolated from day 7 hypothalamic and hippocampal cultures. Gene expression of agouti related peptide (AgRP), POMC, G protein-coupled receptor 101 (GPR101), and Prospero homeobox protein 1 (PROX1) relative to actin is graphed as mean \pm SEM. ${ }^{*} P<0.05$ and ${ }^{* *} P<0.01$ using a Students $t$-test. Data collected from 7 hypothalamic and hippocampal samples from 3 experimental days. (D) Neurons cultured from mice with hypothalamic inducible cre alleles (POMC-CreER and Mchr1-CreER) show the presence of reporter (tdTomato) expression in vitro upon 4-OH tamoxifen treatment. In all images Hoechst stained nuclei are blue and scale bars are $10 \mu \mathrm{m}$.

(Cowley et al., 2001; Gao and van den Pol, 2001; Davidowa et al., 2002, 2003). The ability to culture hypothalamic derived primary neurons that establish and maintain their identity and responsiveness can be utilized for rapidly assessing genetic perturbation and pharmacological responses. The use of MEAs offers the ability to rapidly analyze network effects which can then be followed up using more time consuming and expensive traditional electrophysiological approaches or leadingedge DREADD and optogenetic approaches in vivo (Bernstein and Boyden, 2011; Spira and Hai, 2013; Roth, 2016).

We sought to use these neurons to learn how primary cilia mediated signaling pathways may potentially interact. Dysfunction of primary cilia leads to hyperphagia and obesity in patients with genetic disorders such as BBS and ALMS (Vaisse et al., 2017). Nearly half of neurons and a quarter of glial cells possess ACIII positive primary cilia, similar to what we see in our hippocampal cultures and has been previously reported in cultured hippocampal neurons (Berbari et al., 2007). It is likely that even more cultured cells possess primary cilia, as ACIII is not a universal neuronal cilia marker (Antal et al., 2017). Not only do our cultured hypothalamic neurons possess primary cilia but we also see Mchr1 localize to the cilia in agreement with previous studies (Berbari et al., 2008a,b). The MCH pathway plays a recognized role in feeding behavior (Qu et al., 1996; Chen et al., 2002); however, the role of ciliary localization of Mchr1 is poorly understood. Primary hypothalamic cultures will help 



FIGURE 3 | Hypothalamic specific responses to leptin in vitro. (A) Phosphorylation and nuclear localization of STAT3 (pSTAT3, red) following 30 min leptin (10 nM) treatment. Hoechst stained nuclei are blue and scale bars are $10 \mu \mathrm{m}$. Percent pSTAT3 positive cells in vehicle and leptin treated cultures divided by total number of cells counted is graphed as mean \pm SEM. ${ }^{*} P<0.05$ using a Student's $t$-test. Data collected from 3 hypothalamic samples from 3 experimental days. pSTAT3 positive cells/total vehicle treated hypothalamic nuclei 34/333, pSTAT3 positive cells/total leptin treated hypothalamic nuclei 86/354. (B) Representative traces from one electrode showing the baseline activity and activity after $10 \mathrm{nM}$ leptin addition. The change in firing rate relative to baseline is graphed as mean \pm SEM for vehicle and leptin addition. Each point represents an active electrode. Dashed line indicates two time above and below the average of the vehicle bar. Leptin results in some electrodes displaying decreases (red squares), increases (blue squares), or no change (gray squares) in neuronal activity relative to baseline. Data collected from 4 hypothalamic samples from 3 experimental days.

to address cell biology questions regarding ciliary vs. non-ciliary Mchr1 signaling in relevant cell types.

One well-studied pathway that is coordinated by primary cilia is the hedgehog pathway (Huangfu et al., 2003; Huangfu and Anderson, 2005; Goetz and Anderson, 2010). This pathway is crucial for embryonic development (Szabo et al., 2009) but there may be important roles for the pathway in postmitotic neurons as well. For example, hedgehog pathway components are found in adult hippocampal cells and treatment with hedgehog ligand can increase the excitability of cultured hippocampal neurons (Mitchell et al., 2012). Another study showed that the agonist SAG decreased the firing rate of cultured cortical neurons (Ugbode et al., 2017), further supporting that the hedgehog pathway can influence neuronal activity. In addition to the idea that the hedgehog pathway may alter neuronal firing, there are other studies that led us to hypothesize that it perhaps can influence GPCR signaling in postmitotic cells. For example, GPR161, is critical for proper hedgehog signaling in development (Mukhopadhyay et al., 2013; Pusapati et al., 2018). In addition, conditional deletion of the smoothened gene from olfactory sensory neurons leads to a decrease of odorant GPCR localization to olfactory cilia (Maurya et al., 2017). We are interested in understanding if similar connections exist between hedgehog signaling and cilia GPCRs in neurons. Here we report for the first time that hedgehog pathway agonism can attenuate neuronal responses to $\mathrm{MCH}$. From our data it appears that a subpopulation of cells in hypothalamic cultures respond to $\mathrm{MCH}$ with a decrease in firing rate and that SAG treatment can block that response. Mchr1 is expressed on different cell types in different nuclei of the hypothalamus, some of which include AgRP and POMC neurons within the ARC and several types of neurons within the PVN (Chee et al., 2013). It is unclear which cell type or types are impacted by $\mathrm{MCH}$ and SAG treatment and this will be the focus of future studies.

Activation of the hedgehog pathway involves ligand dependent dynamic localization of both the receptor, patched, and mediator smoothened, out of and into primary cilia (Corbit et al., 2005; Rohatgi et al., 2007). We originally predicted that Mchr1 can also dynamically localize and that SAG treatment may cause it to move out of the cilia, resulting in a dampened response to $\mathrm{MCH}$ following pretreatment with hedgehog agonists. If this were happening, we would have expected to see fewer cilia containing Mchr1 in the SAG treated cultures. However, we did not observe changes in total cilia number, Mchr1 labeled cilia, or cilia length. Therefore, we do not think that dynamic localization of Mchr1 out of the cilia is responsible for the lack of electrophysiological response to ligand following SAG treatment. There are other possibilities for how SAG could be impeding Mchr1 signaling. Proper hedgehog signaling relies on post-translational modifications (Hsia et al., 2015; Arensdorf et al., 2016). It would be interesting if activation of the hedgehog pathway also resulted in post-translational modifications to ciliary GPCRs, like Mchr1, and thus change its downstream signaling. In addition, several GPCRs can 

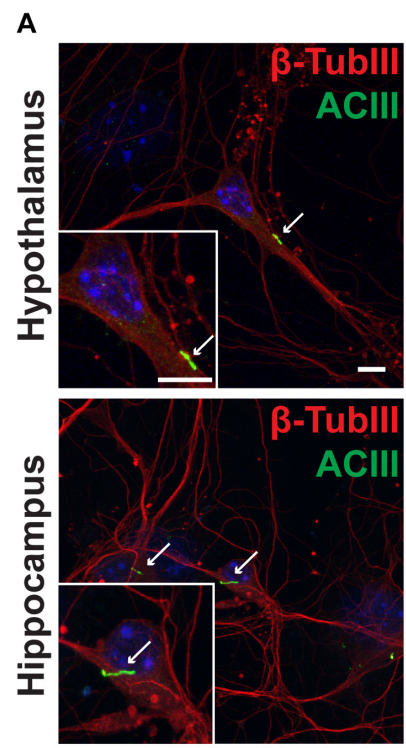

Ciliated

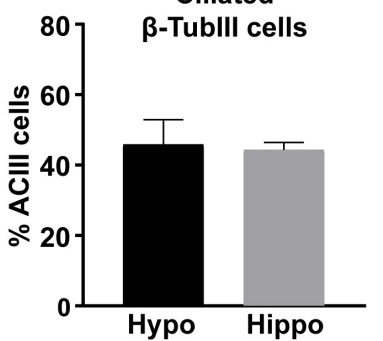

B
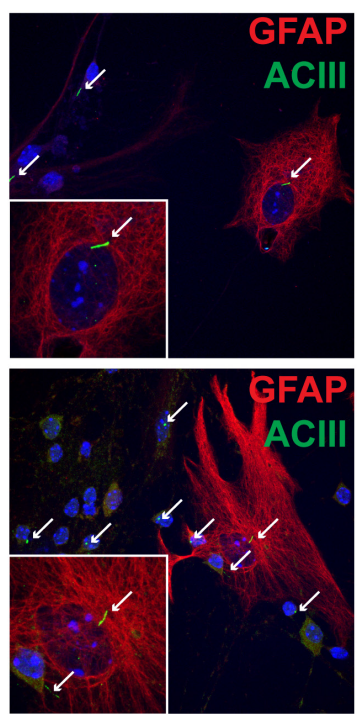

Ciliated

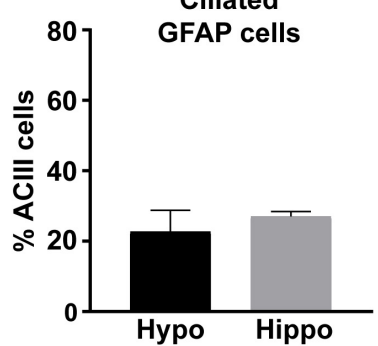

C
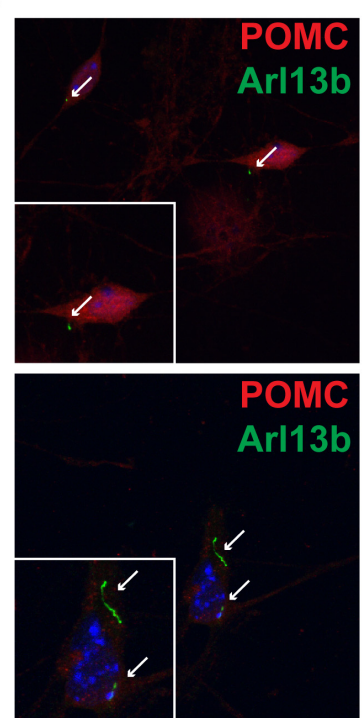

Ciliated

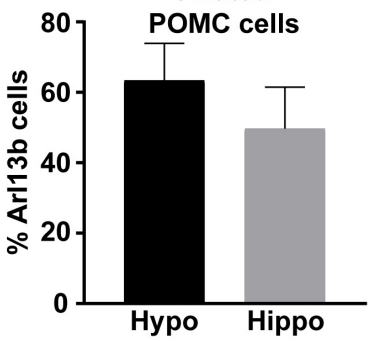

D


Ciliated

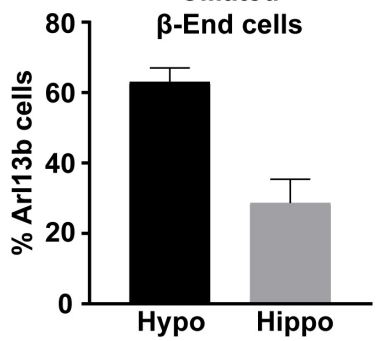

FIGURE 4 | Cultures possess primary cilia. (A) $\beta$-tubulin III ( $\beta$-TubllI, red) stained neurons with adenylyl cyclase III (ACIII, green) positive cilia. (B) Glial fibrillary acidic protein (GFAP, red) stained astrocytes with adenylyl cyclase III (ACIII, green) positive cilia. (C) Proopiomelanocortin (POMC, red) expressing, and (D) $\beta$-endorphin ( $\beta$-End, red) expressing neurons with ADP Ribosylation Factor Like GTPase 13B (Arl13b, green) positive cilia in both hypothalamic and hippocampal derived neurons. Hoechst stained nuclei are blue. Arrows indicate cilia. Scale bars are $10 \mu \mathrm{m}$. Percentage of cilia marker positive cells divided by the total number of associated cell type assessed per hypothalamus or hippocampus is graphed as mean \pm SEM. Data collected from 3 hypothalamic and hippocampal samples from 3 experimental days. $\beta$-tubulin III cells with ACIII positive cilia/total hypothalamic $\beta$-tubulin III positive cells: 152/336, $\beta$-tubulin III cells with ACIII positive cilia/total hippocampal $\beta$-tubulin III positive cells: 160/323, GFAP cells with ACIII positive cilia/total hypothalamic GFAP positive cells: 32/126, GFAP cells with ACIII positive cilia/total hippocampal GFAP positive cells: 42/108, POMC cells with Arl13b positive cilia/total hypothalamic POMC positive cells: 100/166, POMC cells with Arl13b positive cilia/total hippocampal POMC positive cells: 28/57, $\beta$-endorphin cells with Arl13b positive cilia/total hypothalamic $\beta$-endorphin positive cells: 124/203, and $\beta$-endorphin cells with Arl13b positive cilia/total hippocampal $\beta$-endorphin positive cells: $37 / 69$.

biochemically interact including those within the cilium (Green et al., 2012; Gomes et al., 2016). Perhaps when smoothened, itself a seven transmembrane protein, is activated it moves to the cilia and heterodimerizes with Mchr1 causing a change in signaling capabilities. However, our staining results for smoothened upon SAG treatment suggest that there are relatively few smoothened positive cilia (data not shown). It is possible that antibody staining for either Mchrl or smoothened in the cilia is technically limiting due to changes in epitopes of interacting GPCRs. Alternatively, it is also possible that SAG acts on non-Mchr1 expressing cells that then inhibit the firing of Mchr1 neurons. Future studies will involve exploring the mechanism of how hedgehog pathway components may interact or influence not only Mchr1 signaling, but also signaling of other GPCRs known to preferentially localize to primary cilia such as Drd1, Kiss1r, 5-Htr6, Npy5r, Mc4r, and Sstr3 (Hamon et al., 1999; Handel et al., 1999; Brailov et al., 2000; Schulz et al., 2000; Marley and von Zastrow, 2010; Domire et al., 2011; Loktev and Jackson, 2013; Koemeter-Cox et al., 2014; Siljee et al., 2018). Ultimately, experiments addressing the in vivo relevance of any observed interactions are needed.

\section{MATERIALS AND METHODS}

\section{Animals}

Wildtype primary cultures were obtained from C57BL/6J mice from Jackson Laboratories. Cre lines Pomc-CreER (generously provided by Joel K. Elmquist and Chen Liu) and Mchr1CreER have both been previously described (Berglund et al., 2013; Engle et al., 2018). All procedures and approaches were approved by the Institutional Animal Care and Use Committee at Indiana University-Purdue University School of Science, Indianapolis. 
A

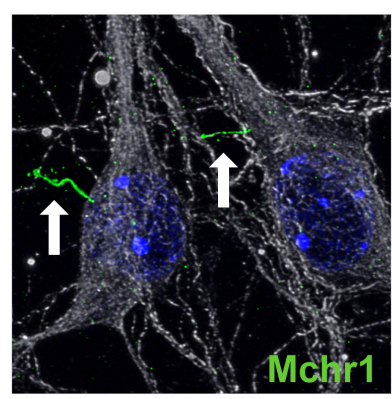

Total

B



E

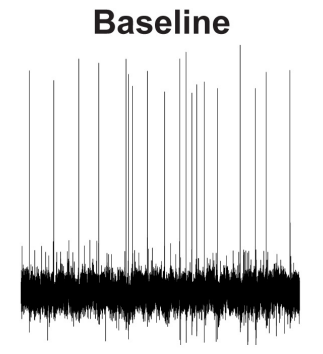

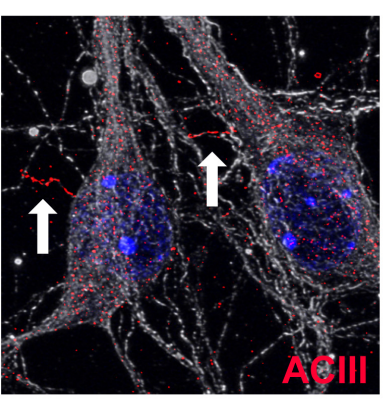

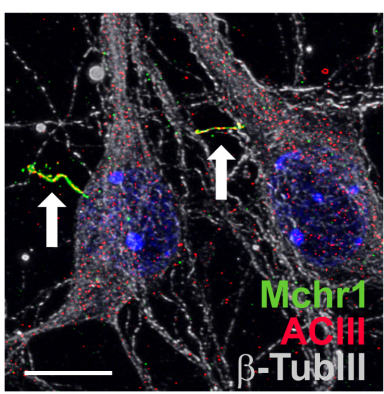

Ciliary Mchr1

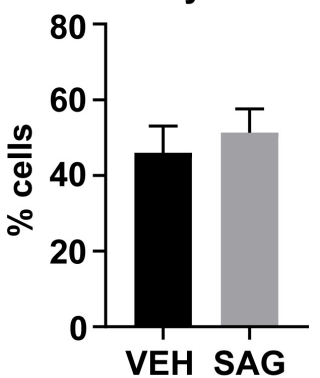

D

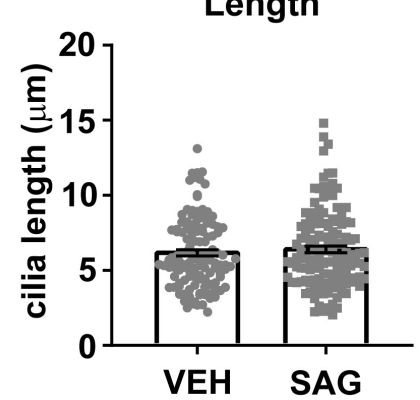



Baseline
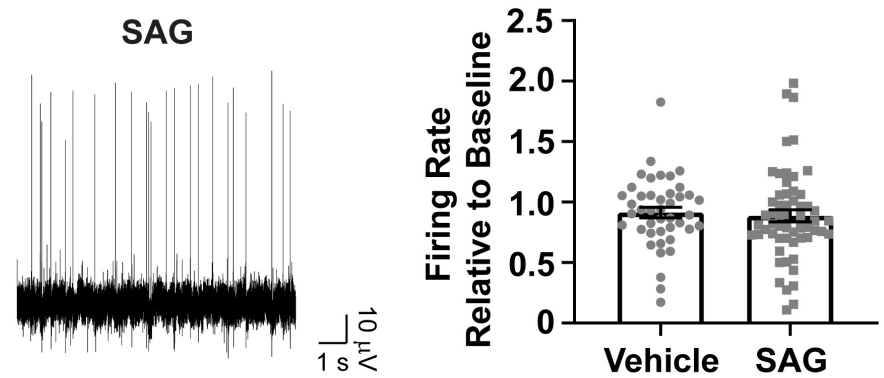

$\mathrm{MCH}$
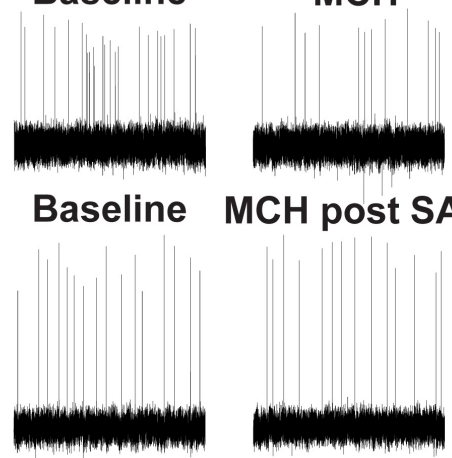

MCH post SAG
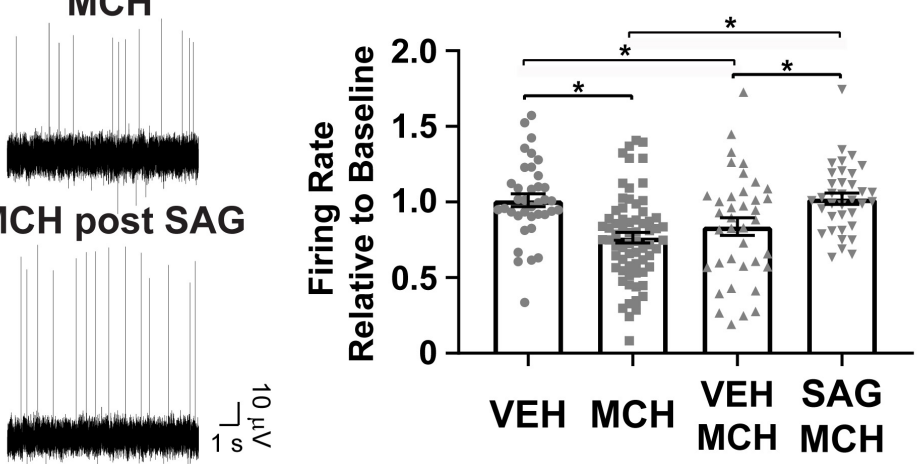

FIGURE 5 | Hedgehog pathway agonism attenuates neuronal response to $\mathrm{MCH}$. (A) Melanin concentrating hormone receptor 1 (Mchr1, green) localizes to primary cilia (ACIII, red) of cultured hypothalamic neurons labeled with $\beta$-tubulin III ( $\beta$-TubIII, gray). Hoechst stained nuclei blue. Scale bar $10 \mu \mathrm{m}$. Arrows indicate cilia. (B) Cilia counts in hypothalamic cultures following $24 \mathrm{~h}$ SAG treatment. Percent cilia positive cells in vehicle and SAG treated cultures is graphed as mean \pm SEM. Vehicle treated hypothalamic cultures: 121 ACIII positive cilia/189 total cells. SAG treated hypothalamic cultures: 127 ACIII positive cilia/189 total cells. (C) Mchr1 positive cilia following SAG treatment. Percent Mchr1 positive cilia in vehicle and SAG treated cultures are graphed as mean \pm SEM. Vehicle treated hypothalamic cultures: 83 Mchr1 positive cilia/121 ACIII positive cilia. SAG treated hypothalamic cultures: 96 Mchr1 positive cilia/127 ACIII positive cilia. (D) Cilia length measurements. Graphed as mean \pm SEM. Outliers were identified and removed using ROUT with $Q=1 \%$. 121 cilia were measured following 24 h vehicle treatment and 127 cilia were measured following $24 \mathrm{~h}$ SAG treatment. (E) Representative traces from one electrode showing baseline activity and activity after addition 400 nM SAG. The change in firing relative to baseline is graphed as mean \pm SEM for vehicle and SAG addition. (F) Representative traces from one electrode showing baseline activity and activity after addition of $1 \mu \mathrm{M} \mathrm{MCH}$ or $\mathrm{MCH}$ following $24 \mathrm{~h}$ pretreatment with $400 \mathrm{nM} \mathrm{SAG}$. The change in firing relative to baseline is graphed as mean $\pm \mathrm{SEM}$ for vehicle and peptide addition. Each point represents an active electrode. ${ }^{*} P<0.05$ using an Ordinary one-way ANOVA with Tukey's multiple comparisons test. Outliers were identified and removed using ROUT with $\mathrm{Q}=1 \%$. All data collected from 3 to 6 hypothalamic samples from 3 to 5 experimental days. 


\section{Primary Neuronal Culture}

Primary neuronal cultures were established as previously reported (Berbari et al., 2007, 2008b). Briefly, on the day of birth, mice were decapitated and the hypothalamus and hippocampus were placed in $0.2 \mathrm{mg} / \mathrm{mL}$ bovine serum albumin (Sigma-Aldrich, St. Louis, MO, United States) in Leibovitz's L15 media (L15/BSA) (Gibco, Gaithersburgh, MD, United States). The tissue was then transferred to L15/BSA with $0.375 \mathrm{mg} / \mathrm{mL}$ papain (Sigma-Aldrich) under $95 \% \quad \mathrm{O}_{2} / 5 \% \quad \mathrm{CO}_{2}$ gas for $10 \mathrm{~min}$ at $37^{\circ} \mathrm{C}$. This was followed by two washes in Earle's Minimal Essential Medium (Gibco) containing 5\% fetal bovine serum (Gibco), 5\% horse serum (Gibco), $400 \mu \mathrm{M}$ glutamax (Gibco), $16.65 \mathrm{mM}$ glucose (Sigma-Aldrich), and $2.5 \mu \mathrm{l} / \mathrm{ml}$ insulin/transferrin/selenite (Sigma-Aldrich), collectively called M5-5 media. The cells were mechanically dissociated by trituration through a series of three flame polished Pasteur pipettes with each subsequent pipette having a smaller bore size. The cells were then spun down at $120 \times g$ for $5 \mathrm{~min}$ at room temperature, pellets were resuspended in M5-5 media ( $2 \mathrm{~mL} /$ hypothalamus, $3 \mathrm{~mL} /$ hippocampus), and plated on poly-L-Lysine (Sigma-Aldrich) coated coverslips in 24 well plates. Hypothalamic cells were plated on two coverslips per animal at a starting density of 300,000 cells/well while hippocampal cells were distributed on three coverslips per animal at a starting density of 400,000 cells/well. Two days later, half of M5-5 media was removed and replaced with $1 \mathrm{~mL}$ of Neurobasal media (Gibco) supplemented with $2 \%$ B27 supplement (Gibco), $2.5 \mu \mathrm{l} / \mathrm{ml}$ insulin/transferrin/selenite, $500 \mu \mathrm{M}$ glutamax, and $1 \mu \mathrm{l} / \mathrm{ml}$ gentamycin (Gibco) for immunostaining approaches. The media was supplemented with $10 \mu \mathrm{M}$ cytosine arabinofuranoside (ARA-C, Sigma-Aldrich) to prevent cell proliferation. Cells were incubated at $37^{\circ} \mathrm{C}$ and $5 \%$ $\mathrm{CO}_{2}$ for 7-10 days for immunofluorescence studies or 10-14 days for electrophysiological studies.

\section{CreER Induction}

To induce CreER in vitro, neurons were treated with $0.5 \mu \mathrm{M}$ 4-hydroxytamoxifen (Sigma Aldrich) (Danielian et al., 1998) at 5 days in culture and fixed on day 7 for immunofluorescence as described below.

\section{Immunofluorescence}

Immunofluorescence was performed as previously reported (Berbari et al., 2008a). Briefly, after 7-10 days in culture, cells were fixed for $10 \mathrm{~min}$ at room temperature with a solution of either $4 \%$ paraformaldehyde (PFA;Affymetrix/USB, Cleveland, $\mathrm{OH}$, United States) $/ 10 \%$ sucrose (Dot Scientific Inc, Burton, MI, United States) or when staining with the Mchrl antibody 4\% PFA/Histochoice (Sigma-Aldrich). Fixation was followed by incubation in 100\% methanol (Thermo Fisher Scientific) for $15 \mathrm{~min}$ at $-20^{\circ} \mathrm{C}$. The cells were then permeabilized and blocked with $0.1 \%$ Triton X-100 (Thermo Fisher Scientific) in PBS for 6 min and blocked in PBS containing 1\% BSA, $0.1 \%$ Triton $\mathrm{X}-100,2 \%$ donkey serum, and $0.02 \%$ sodium azide (Dot Scientific) for at least half an hour. Cells were then incubated in primary antibodies in blocking solution overnight at $4^{\circ} \mathrm{C}$. Following 3 five-minute washes in PBS, the cells were incubated with secondary antibodies in blocking solution for $1.5 \mathrm{~h}$ at room temperature. Nuclei were stained with Hoechst 33342 (Thermo Fisher Scientific, Waltham, MA, United States) and cells were mounted on slides using 1,4diazabicyclo[2.2.2] octane (DABCO) and sealed with nail polish or ProLong Diamond (Invitrogen, Carlsbad, CA, United States). Primary cilia were stained with either Arl13b (mouse, 1:300; UC Davis/NIH NeuroMab Facility, Davis, CA, United States 75287), ACIII (rabbit, 1:500; Santa Cruz Biotechnology, Dallas, TX, United States sc-588), ACIII (rabbit, 1:5000; EnCor Biotechnology, Gainsville, FL, United States RPCA_ACIII), or Mchr1 (goat, 1:250; Santa Cruz Biotechnology sc-5534). Neurons were detected using $\beta$-endorphin (rabbit, 1:250; Phoenix Pharmaceuticals, Burlingame, CA, United States H-022-33), POMC (rabbit, 1:250; Phoenix Pharmaceuticals H-029-30), or $\beta$-tubulin III (mouse, 1:1000; Sigma Life Sciences, St. Louis, MO, United States T8660) antibodies. Glial cells were stained with GFAP (mouse, 1:1000; Sigma Life Sciences G3893) antibody. Induction of phosphorylation of STAT3 was identified by staining cells with pSTAT3 (mouse, 1:200; Thermo Fisher Scientific MA5-15193). The secondary antibodies used were donkey antimouse Alexa 488 (Thermo Fisher Scientific, Waltham, MA, United States A21202), donkey anti-rabbit Alexa 546 (Thermo Fisher Scientific A10040), donkey anti-rabbit Alexa 488 (Thermo Fisher Scientific A21206), donkey anti-mouse Alexa 546 (Thermo Fisher Scientific A10036), donkey anti-goat Alexa 546 (Thermo Fisher Scientific A11056), and donkey anti-mouse Alexa 647 (Thermo Fisher Scientific A31571) at 1:1000 dilution. Control experiments included secondary antibody only.

\section{Imaging}

Cultured neurons were imaged with a Leica SP8 confocal microscope in resonant scanning mode using a $63 \times$ NA 1.4 objective. For all images collected, contrast and intensity were adjusted using ImageJ. For counting cells, samples were imaged with a Nikon $90 \mathrm{i}$ epifluorescent microscope equipped with a Hamamatsu Orca Flash4-LT CMOS camera, SOLA-SE II LED illuminator.

\section{Cell and Cilia Counts}

Fields were randomly selected from hypothalamic and hippocampal cultures. The total number of positively stained cells were counted and the results were tabulated as percentage by dividing by the total number of cells selected. ImageJ was used to measure the intensity of pSTAT3 staining in the nucleus of vehicle and leptin treated cultures. Any nuclei with a value twice as high as average background intensity was counted as pSTAT3 positive.

\section{Cilia Length Measurements}

Z-stack images of cilia were collected with a Leica SP8 confocal microscope in resonant scanning mode using a $63 \times$ NA 1.4 objective. Images were collected in $0.2 \mu \mathrm{m}$ thick slices and pixel size for each stack was kept at $45 \mathrm{~nm} \times 45 \mathrm{~nm}$. The images were then deconvolved using Lightning Deconvolution module in Leica Application Software (LAS X). Cilia lengths 
were then measured in a three-dimensional space using $3 \mathrm{D}$ measurement module in LAS X by manually tracing along the lengths of the cilia.

\section{RT-qPCR}

RNA was isolated using the RNeasy Micro Kit (Qiagen, Venlo, Netherlands) and then reverse transcribed into cDNA using the Quantitect Reverse Transcription Kit (Qiagen). Reactions were performed on an Applied Biosystems QuantStudio 7 RealTime PCR System (Applied Biosystems, South San Francisco, CA, United States), CT values were normalized to $\beta$-actin, and relative expression was calculated by the $\Delta \Delta \mathrm{CT}$ method. Assays-onDemand Gene expression probes (Applied Biosystems) were as follows: AGRP Mm00435874_m1, POMC Mm01251072_g1, GPR101 Mm01296083_m1, PRDM12 Mm01324474_m1, and Prox1 Mm00435969_m1.

\section{MEA Recordings}

Primary neurons were prepared as above with the entire hypothalamus being resuspended in $1 \mathrm{~mL}$ M5-5 media and plated on poly-L-lysine treated MEAs (60MEA200/30iR-Ti, Multichannel Systems, Reutlingen, Germany). Lids were made to cover the arrays from fluorinated ethylene-propylene (FEP) film (Scientific Commodities, INC. catalog \# BB3090-1-24, Lake Havasu City, AZ, United States) attached to plastic cylinders with $\mathrm{O}$-rings. MEAs were incubated at $37^{\circ} \mathrm{C}$ in $5 \%$ $\mathrm{CO}_{2}$ for $48 \mathrm{hrs}$ before the M5-5 plating media was replaced with BrainPhys Neuronal Media (STEMCELL Technologies, Vancouver, BC, Canada) (Bardy et al., 2015). Every $10 \mathrm{ml}$ of the media was supplemented with $200 \mu 1$ NeuroCult SM1 neuronal supplement (STEMCELL Technologies), $100 \mu 1$ N2 SupplementA (STEMCELL Technologies), $20 \mathrm{ng} / \mathrm{ml}$ Recombinant Human Brain-Derived Neurotrophic Factor (STEMCELL Technologies), 20 ng/ml Recombinant Human Glial-Derived Neurotrophic Factor (STEMCELL Technologies), $1 \mathrm{mM}$ dibutyryl cAMP (Santa Cruz Biotechnology), 200 nM ascorbic acid (STEMCELL Technologies), $1 \mu \mathrm{L} / \mathrm{mL}$ gentamycin and $10 \mu \mathrm{M}$ ARA-C. A MEA2100-System (Multichannel Systems) with a headstage maintained at $37^{\circ} \mathrm{C}$ was used to acquire extracellular recordings from the cultures 10 days after they were plated on MEAs. Data was acquired from 60 electrode channels at a sampling frequency of $50 \mathrm{kHz}$ per channel using MC_Rack and Multi-Channel Experimenter software (Multichannel Systems). Spontaneous field potentials were recorded during the addition of vehicle or drug. Media was removed from the MEAs for vehicle and drug dilutions. Baseline activity was recorded for several minutes in the presence of vehicle. While continuing to record, the following drugs were added to individual MEAs: leptin (10 nM in Tris; PeproTech Inc, Rocky Hill NJ; Cat. \# 450-31 or R\&D Systems, Minneapolis, MN, United States; Cat. \# 4980B01M), $\mathrm{MCH}\left(1 \mu \mathrm{M}\right.$ in $\mathrm{H}_{2} \mathrm{O}$; Tocris Bioscience, Minneapolis, $\mathrm{MN}$, United States; Cat. \# 3806100U), SAG (400 nM in DMSO). For priming experiments, cells were pretreated with $400 \mathrm{nM}$ SAG $24 \mathrm{~h}$ before measuring activity in response to $\mathrm{MCH}$. Action potential frequency was analyzed using a combination of MEA Tools within MEA Viewer (Bridges et al., 2018) and MATLAB scripting ${ }^{1}$. Spike data was extracted using MEA Tools, including spikes exceeding $\pm 5 \mathrm{X}$ standard deviation of signal following a Butterworth filter. Data was then organized into a standardized matrix structure based on electrode layout, active electrodes, and timepoint before analysis. Electrodes were considered active when the baseline firing rate was greater than $0.5 \mathrm{~Hz}$. Normalized firing rate was calculated by dividing the firing rate in the presence of drug by the baseline firing rate. Baseline firing rate was determined from the minute immediately preceding drug addition. Firing in the presence of drug was determined over a $60 \mathrm{~s}$ window during peak drug response ( $3 \mathrm{~min}$ following $\mathrm{MCH}$ addition, 4 min following leptin and SAG addition).

\section{Statistical Analysis}

All statistical tests were performed using GraphPad Prism. All statistically significant observations are noted in the figures and specific tests used are within the legends.

\section{DATA AVAILABILITY}

All datasets generated for this study are included in the manuscript and/or the supplementary files.

\section{ETHICS STATEMENT}

All experimental procedures were approved by the Institutional Animal Care and Use Committee (IACUC) at the Indiana University - Purdue University Indianapolis.

\section{AUTHOR CONTRIBUTIONS}

$\mathrm{RB}, \mathrm{SE}$, and $\mathrm{NB}$ conceived and designed the experiments. $\mathrm{RB}, \mathrm{SE}, \mathrm{PA}, \mathrm{LW}, \mathrm{AB}$, and NB performed the experiments. All authors analyzed the data. TC and NB contributed to reagents, materials, and analysis tools. $\mathrm{RB}, \mathrm{SE}, \mathrm{JR}$, and $\mathrm{NB}$ wrote the manuscript.

\section{FUNDING}

This work was supported by the NIH National Institute of Diabetes and Digestive and Kidney Diseases (R01 DK114008) and the American Heart Association Grant \#18PRE34020122/Ruchi Bansal/2018.

\section{ACKNOWLEDGMENTS}

We thank Dr. Joel Elmquist and Dr. Chen Liu for generously providing us with POMC-CreER mice. We acknowledge Dr. Benjamin Perrin and Dr. Lata Balakrishnan for critical review of the manuscript.

\footnotetext{
${ }^{1}$ https://github.com/Lswhiteh/MEA_Data_Analysis
} 


\section{REFERENCES}

Andermann, M. L., and Lowell, B. B. (2017). Toward a Wiring diagram understanding of appetite control. Neuron 95, 757-778. doi: 10.1016/j.neuron. 2017.06.014

Antal, M. C., Bénardais, K., Samama, B., Auger, C., Schini-Kerth, V., Ghandour, S., et al. (2017). Adenylate cyclase type iii is not a ubiquitous marker for all primary cilia during development. PLoS One 12:e170756. doi: 10.1371/journal. pone.0170756

Arensdorf, A. M., Marada, S., and Ogden, S. K. (2016). Smoothened regulation: a tale of two signals. Trends Pharmacol. Sci. 37, 62-72. doi: 10.1016/j.tips.2015. 09.001

Banks, A. S., Davis, S. M., Bates, S. H., and Myers, M. G. Jr. (2000). Activation of downstream signals by the long form of the leptin receptor. J. Biol. Chem. 275, 14563-14572. doi: 10.1074/jbc.275.19.14563

Bardy, C., van den Hurk, M., Eames, T., Marchand, C., Hernandez, R. V., Kellogg, M., et al. (2015). Neuronal medium that supports basic synaptic functions and activity of human neurons in vitro. Proc. Natl. Acad. Sci. U.S.A. 112, E2725-E2734.

Berbari, N. F., Bishop, G. A., Askwith, C. C., Lewis, J. S., and Mykytyn, K. (2007). Hippocampal neurons possess primary cilia in culture. J. Neurosci. Res. 85, 1095-1100. doi: 10.1002/jnr.21209

Berbari, N. F., Johnson, A. D., Lewis, J. S., Askwith, C. C., and Mykytyn, K. (2008a). Identification of ciliary localization sequences within the third intracellular loop of G protein-coupled receptors. Mol. Biol. Cell 19, 1540-1547. doi: 10.1091/mbc. E07-09-0942

Berbari, N. F., Lewis, J. S., Bishop, G. A., Askwith, C. C., and Mykytyn, K. (2008b). Bardet-biedl syndrome proteins are required for the localization of $\mathrm{G}$ proteincoupled receptors to primary cilia. Proc. Natl. Acad. Sci. U.S.A. 105, 4242-4246. doi: 10.1073/pnas.0711027105

Berbari, N. F., O'Connor, A. K., Haycraft, C. J., and Yoder, B. K. (2009). The primary cilium as a complex signaling center. Curr. biol. 19, R526-R535. doi: 10.1016/j.cub.2009.05.025

Berbari, N. F., Pasek, R. C., Malarkey, E. B., Yazdi, S. M., McNair, A. D., Lewis, W. R., et al. (2013). Leptin resistance is a secondary consequence of the obesity in ciliopathy mutant mice. Proc. Natl. Acad. Sci. U.S.A. 110, 7796-7801. doi: 10.1073/pnas.1210192110

Berglund, E. D., Liu, C., Sohn, J. W., Liu, T., Kim, M. H., Lee, C. E., et al. (2013) Serotonin 2C receptors in pro-opiomelanocortin neurons regulate energy and glucose homeostasis. J. Clin. Invest. 123, 5061-5070. doi: 10.1172/JCI7 0338

Bernstein, J. G., and Boyden, E. S. (2011). Optogenetic tools for analyzing the neural circuits of behavior. Trends Cogn. Sci. 15, 592-600. doi: 10.1016/j.tics.2011. 10.003

Besschetnova, T. Y., Kolpakova-Hart, E., Guan, Y., Zhou, J., Olsen, B. R., and Shah, J. V. (2010). Identification of signaling pathways regulating primary cilium length and flow-mediated adaptation. Curr. Biol. 20, 182-187. doi: 10.1016/j. cub.2009.11.072

Bishop, G. A., Berbari, N. F., Lewis, J., and Mykytyn, K. (2007). Type III adenylyl cyclase localizes to primary cilia throughout the adult mouse brain. J. Comp. Neurol. 505, 562-571. doi: 10.1002/cne.21510

Bittencourt, J. C., Presse, F., Arias, C., Peto, C., Vaughan, J., Nahon, J. L., et al. (1992). The melanin-concentrating hormone system of the rat brain: an immuno- and hybridization histochemical characterization. J. Comp. Neurol. 319, 218-245. doi: 10.1002/cne.903190204

Brailov, I., Bancila, M., Brisorgueil, M. J., Miquel, M. C., Hamon, M., and Verge, D. (2000). Localization of 5-HT(6) receptors at the plasma membrane of neuronal cilia in the rat brain. Brain Res. 872, 271-275. doi: 10.1016/s0006-8993(00) 02519-1

Bridges, D. C., Tovar, K. R., Wu, B., Hansma, P. K., and Kosik, K. S. (2018). MEA viewer: a high-performance interactive application for visualizing electrophysiological data. PLoS One 13:e0192477. doi: 10.1371/journal.pone. 0192477

Caspary, T., Larkins, C. E., and Anderson, K. V. (2007). The graded response to Sonic hedgehog depends on cilia architecture. Dev. Cell 12, 767-778. doi: 10.1016/j.devcel.2007.03.004

Chee, M. J., Pissios, P., and Maratos-Flier, E. (2013). Neurochemical characterization of neurons expressing melanin-concentrating hormone receptor 1 in the mouse hypothalamus. J. Comp. Neurol. 521, 2208-2234. doi: $10.1002 /$ cne. 23273

Chen, Y., Hu, C., Hsu, C. K., Zhang, Q., Bi, C., Asnicar, M., et al. (2002). Targeted disruption of the melanin-concentrating hormone receptor-1 results in hyperphagia and resistance to diet-induced obesity. Endocrinology 143, 2469-2477. doi: 10.1210/en.143.7.2469

Corbit, K. C., Aanstad, P., Singla, V., Norman, A. R., Stainier, D. Y., and Reiter, J. F. (2005). Vertebrate smoothened functions at the primary cilium. Nature 437, 1018-1021. doi: 10.1038/nature04117

Cowley, M. A., Smart, J. L., Rubinstein, M., Cerdán, M. G., Diano, S., Horvath, T. L., et al. (2001). Leptin activates anorexigenic POMC neurons through a neural network in the arcuate nucleus. Nature 411:480. doi: 10.1038/3507 8085

Danielian, P. S., Muccino, D., Rowitch, D. H., Michael, S. K., and McMahon, A. P. (1998). Modification of gene activity in mouse embryos in utero by a tamoxifen-inducible form of cre recombinase. Curr. biol. 8, 1323-1326.

Davenport, J. R., Watts, A. J., Roper, V. C., Croyle, M. J., van Groen, T., Wyss, J. M., et al. (2007). Disruption of intraflagellar transport in adult mice leads to obesity and slow-onset cystic kidney disease. Curr. biol. 17, 1586-1594. doi: 10.1016/j.cub.2007.08.034

Davidowa, H., Li, Y., and Plagemann, A. (2002). Hypothalamic ventromedial and arcuate neurons of normal and postnatally overnourished rats differ in their responses to melanin-concentrating hormone. Regul. pept. 108, 103-111. doi: 10.1016/s0167-0115(02)00153-2

Davidowa, H., Li, Y., and Plagemann, A. (2003). Altered responses to orexigenic (AGRP, MCH) and anorexigenic (alpha-MSH, CART) neuropeptides of paraventricular hypothalamic neurons in early postnatally overfed rats. Eur. J. Neurosci. 18, 613-621. doi: 10.1046/j.1460-9568.2003.02789.x

Desy, L., and Pelletier, G. (1978). Immunohistochemical localization of alphamelanocyte stimulating hormone (alpha-MSH) in the human hypothalamus. Brain Res. 154, 377-381. doi: 10.1016/0006-8993(78)90709-6

Domire, J. S., Green, J. A., Lee, K. G., Johnson, A. D., Askwith, C. C., and Mykytyn, K. (2011). Dopamine receptor 1 localizes to neuronal cilia in a dynamic process that requires the bardet-biedl syndrome proteins. Cell. Mol. Life Sci. 68, 2951-2960. doi: 10.1007/s00018-010-0603-4

Engle, S. E., Antonellis, P. J., Whitehouse, L. S., Bansal, R., Emond, M. R., Jontes, J. D., et al. (2018). A creer mouse to study melanin concentrating hormone signaling in the developing brain. Genesis 56:e23217. doi: 10.1002/dvg. 23217

Gao, X. B., and van den Pol, A. N. (2001). Melanin concentrating hormone depresses synaptic activity of glutamate and GABA neurons from rat lateral hypothalamus. J. Physiol. 533, 237-252. doi: 10.1111/j.1469-7793.2001.0237b.x

Goetz, S. C., and Anderson, K. V. (2010). The primary cilium: a signalling centre during vertebrate development. Nat. Rev. Genet. 11, 331-344. doi: 10.1038/ $\operatorname{nrg} 2774$

Gomes, I., Ayoub, M. A., Fujita, W., Jaeger, W. C., Pfleger, K. D. G., and Devi, L. A. (2016). G protein-coupled receptor heteromers. Annu. Rev. Pharmacol. Toxicol. 56, 403-425. doi: 10.1146/annurev-pharmtox-011613-135952

Green, J. A., Gu, C., and Mykytyn, K. (2012). Heteromerization of ciliary g proteincoupled receptors in the mouse brain. PLoS One 7:e46304. doi: 10.1371/journal. pone.0046304

Guo, D.-F., Cui, H., Zhang, Q., Morgan, D. A., Thedens, D. R., Nishimura, D., et al. (2016). The bbsome controls energy homeostasis by mediating the transport of the leptin receptor to the plasma membrane. PLoS Genet. 12:e1005890. doi: 10.1371/journal.pgen.1005890

Hamamoto, A., Yamato, S., Katoh, Y., Nakayama, K., Yoshimura, K., Takeda, S., et al. (2016). Modulation of primary cilia length by melanin-concentrating hormone receptor 1. Cell. Signal. 28, 572-584. doi: 10.1016/j.cellsig.2016.02.018

Hamon, M., Doucet, E., Lefevre, K., Miquel, M. C., Lanfumey, L., Insausti, R., et al. (1999). Antibodies and antisense oligonucleotide for probing the distribution and putative functions of central 5-HT6 receptors. Neuropsychopharmacology 21, 68s-76s. doi: 10.1016/s0893-133x(99)00044-5

Handel, M., Schulz, S., Stanarius, A., Schreff, M., Erdtmann-Vourliotis, M., Schmidt, H., et al. (1999). Selective targeting of somatostatin receptor 3 to neuronal cilia. Neuroscience 89, 909-926. doi: 10.1016/s0306-4522(98)00354-6

Hsia, E. Y. C., Gui, Y., and Zheng, X. (2015). Regulation of Hedgehog signaling by ubiquitination. Front. Biol. 10, 203-220. doi: 10.1007/s11515-0151343-5 
Huangfu, D., and Anderson, K. V. (2005). Cilia and Hedgehog responsiveness in the mouse. Proc. Natl. Acad. Sci. U.S.A. 102, 11325-11330. doi: 10.1073/pnas. 0505328102

Huangfu, D., Liu, A., Rakeman, A. S., Murcia, N. S., Niswander, L., and Anderson, K. V. (2003). Hedgehog signalling in the mouse requires intraflagellar transport proteins. Nature 426, 83-87. doi: 10.1038/nature02061

Koemeter-Cox, A. I., Sherwood, T. W., Green, J. A., Steiner, R. A., Berbari, N. F., Yoder, B. K., et al. (2014). Primary cilia enhance kisspeptin receptor signaling on gonadotropin-releasing hormone neurons. Proc. Natl. Acad. Sci. U.S.A. 111, 10335-10340. doi: 10.1073/pnas.1403286111

Loktev, A. V., and Jackson, P. K. (2013). Neuropeptide Y family receptors traffic via the bardet-biedl syndrome pathway to signal in neuronal primary cilia. Cell Reports 5, 1316-1329. doi: 10.1016/j.celrep.2013.11.011

Marley, A., and von Zastrow, M. (2010). DISC1 regulates primary cilia that display specific dopamine receptors. PLoS One 5:e10902. doi: 10.1371/journal.pone. 0010902

Maurya, D. K., Bohm, S., and Alenius, M. (2017). Hedgehog signaling regulates ciliary localization of mouse odorant receptors. Proc. Natl. Acad. Sc.i U.S.A. 114, E9386-E9394. doi: 10.1073/pnas.1708321114

Mitchell, N., Petralia, R. S., Currier, D. G., Wang, Y. X., Kim, A., Mattson, M. P., et al. (2012). Sonic hedgehog regulates presynaptic terminal size, ultrastructure and function in hippocampal neurons. J. Cell Sci. 125, 4207-4213. doi: 10.1242/ jcs. 105080

Mukhopadhyay, S., Wen, X., Ratti, N., Loktev, A., Rangell, L., Scales, S. J., et al. (2013). The ciliary G-protein-coupled receptor Gpr161 negatively regulates the Sonic hedgehog pathway via cAMP signaling. Cell 152, 210-223. doi: 10.1016/j. cell.2012.12.026

Mykytyn, K., Mullins, R. F., Andrews, M., Chiang, A. P., Swiderski, R. E., Yang, B., et al. (2004). Bardet-biedl syndrome type 4 (BBS4)-null mice implicate Bbs4 in flagella formation but not global cilia assembly. Proc. Natl. Acad. Sci. U.S.A. 101, 8664-8669. doi: 10.1073/pnas.0402354101

Pissios, P., Trombly, D. J., Tzameli, I., and Maratos-Flier, E. (2003). Melaninconcentrating hormone receptor 1 activates extracellular signal-regulated kinase and synergizes with G(s)-coupled pathways. Endocrinology 144, 35143523. doi: 10.1210/en.2002-0004

Praetorius, H. A., and Spring, K. R. (2001). Bending the MDCK cell primary cilium increases intracellular calcium. J. Membr. Biol. 184, 71-79. doi: 10.1007/ s00232-001-0075-4

Pusapati, G. V., Kong, J. H., Patel, B. B., Gouti, M., Sagner, A., Sircar, R., et al. (2018). G protein-coupled receptors control the sensitivity of cells to the morphogen Sonic Hedgehog. Sci. Signal. 11:eaao5749. doi: 10.1126/scisignal. aao5749

Qu, D., Ludwig, D. S., Gammeltoft, S., Piper, M., Pelleymounter, M. A., Cullen, M. J., et al. (1996). A role for melanin-concentrating hormone in the central regulation of feeding behaviour. Nature 380, 243-247. doi: 10.1038/380243a0

Rohatgi, R., Milenkovic, L., and Scott, M. P. (2007). Patched1 regulates hedgehog signaling at the primary cilium. Science $317,372-376$. doi: $10.1126 /$ science. 1139740
Roth, B. L. (2016). DREADDs for Neuroscientists. Neuron 89, 683-694. doi: 10. 1016/j.neuron.2016.01.040

Schulz, S., Handel, M., Schreff, M., Schmidt, H., and Hollt, V. (2000). Localization of five somatostatin receptors in the rat central nervous system using subtypespecific antibodies. J. Physiol. Paris 94, 259-264. doi: 10.1016/s0928-4257(00) 00212-6

Schwartz, M. W., Woods, S. C., Porte, D. Jr., Seeley, R. J., and Baskin, D. G. (2000). Central nervous system control of food intake. Nature 404:661. doi: $10.1038 / 35007534$

Siljee, J. E., Wang, Y., Bernard, A. A., Ersoy, B. A., Zhang, S., Marley, A., et al. (2018). Subcellular localization of MC4R with ADCY3 at neuronal primary cilia underlies a common pathway for genetic predisposition to obesity. Nat. Genet. 50, 180-185. doi: 10.1038/s41588-017-0020-9

Singla, V., and Reiter, J. F. (2006). The primary cilium as the cell's antenna: signaling at a sensory organelle. Science 313, 629-633. doi: 10.1126/science.1124534

Spira, M. E., and Hai, A. (2013). Multi-electrode array technologies for neuroscience and cardiology. Nature nanotechnology 8, 83-94. doi: 10.1038/ nnano.2012.265

Szabo, N. E., Zhao, T., Cankaya, M., Theil, T., Zhou, X., and AlvarezBolado, G. (2009). Role of neuroepithelial Sonic hedgehog in hypothalamic patterning. J. Neurosci. 29, 6989-7002. doi: 10.1523/JNEUROSCI.1089-09. 2009

Tomoshige, S., Kobayashi, Y., Hosoba, K., Hamamoto, A., Miyamoto, T., and Saito, Y. (2017). Cytoskeleton-related regulation of primary cilia shortening mediated by melanin-concentrating hormone receptor 1. Gen. Comp. Endocrinol. 253, 44-52. doi: 10.1016/j.ygcen.2017.08.021

Ugbode, C. I., Smith, I., Whalley, B. J., Hirst, W. D., and Rattray, M. (2017). Sonic hedgehog signalling mediates astrocyte crosstalk with neurons to confer neuroprotection. J. Neurochem. 142, 429-443. doi: 10.1111/jnc. 14064

Vaisse, C., Reiter, J. F., and Berbari, N. F. (2017). Cilia and obesity. Cold Spring Harb. Perspect. Biol. 9:a028217. doi: 10.1101/cshperspect.a028217

Yao, P. J., Petralia, R. S., Ott, C., Wang, Y. X., Lippincott-Schwartz, J., and Mattson, M. P. (2015). Dendrosomatic sonic hedgehog signaling in hippocampal neurons regulates axon elongation. J Neurosci. 35, 16126-16141. doi: 10.1523/ JNEUROSCI.1360-15.2015

Conflict of Interest Statement: The authors declare that the research was conducted in the absence of any commercial or financial relationships that could be construed as a potential conflict of interest.

Copyright (c) 2019 Bansal, Engle, Antonellis, Whitehouse, Baucum, Cummins, Reiter and Berbari. This is an open-access article distributed under the terms of the Creative Commons Attribution License (CC BY). The use, distribution or reproduction in other forums is permitted, provided the original author(s) and the copyright owner(s) are credited and that the original publication in this journal is cited, in accordance with accepted academic practice. No use, distribution or reproduction is permitted which does not comply with these terms. 